\title{
Delay effect of switch-on in a supertwisted nematic cell
}

\author{
Fuzi Yang ${ }^{\text {a) }}$ and Youmei Dong \\ Key Lab of Organic Optoelectronics \& Molecular Engineering of Ministry of Education, Beijing 100084, \\ People's Republic of China and Liquid Crystal Research Centre, Chemistry Department, \\ Tsinghua University, Beijing 100084, People's Republic of China \\ L. Z. Ruan and J. R. Sambles \\ Thin Film Photonics, School of Physics, University of Exeter, Exeter, EX4 4QL, United Kingdom
}

(Received 27 February 2004; accepted 24 September 2004)

\begin{abstract}
By using a convergent beam system and the fully leaky guided mode technique the switch-on dynamics of an $180^{\circ}$ supertwisted nematic have been studied. Using the Ericksen-Leslie theory and analyzing the guided mode data taken from the cell, the director structure in the cell at different times during switch-on is obtained. For three different applied voltages it is found that the switch-on time is strongly dependent on the applied field - the higher voltage corresponds to faster switching, with no evidence of backflow. A delay at the beginning of the switch-on process has been found and explored for different applied fields. This leads to a suggestion for increasing the switch-on speed of such devices by 25\%. (C) 2004 American Institute of Physics. [DOI: 10.1063/1.1826240]
\end{abstract}

The supertwisted nematic device (the director twist angle in the cell is more than $90^{\circ}$ ) (STN) has been widely used in portable and notebook computer displays. This is primarily because this device has wider viewing angle and faster electro-optic response, which greatly overcome the disadvantages of the twisted nematic (TN) cell, and is very suitable for high information content displays. The dynamics during switching are important. The Ericksen-Leslie theory, ${ }^{1-3}$ developed in the 1960 's, later simplified by Berreman and van Doorn, ${ }^{4,5}$ is the foundational work concerning dynamic effects in nematic liquid crystal. This theory is still very widely used. There also exist a number of experimental studies $^{6-8}$ on liquid crystal dynamics, including the most significant experiments confirming the theoretically predicted "backflow" in a TN cell during switch-off from a strong external electric field. ${ }^{6,7}$ However, almost all the experiments ${ }^{6-9}$ have been based on simple transmission or reflection observations, and as such the signals from the liquid crystal cell are an integrated response, that is, the changes of the director profile in the cell during the switching process cannot be examined in detail. Only recently, using a convergent-beam optical guided-wave technique ${ }^{10-13}$ have the details of the director profile in liquid crystal cells during switching been unambiguously obtained.

In this work the convergent beam system is used to investigate the switch-on dynamics of a $180^{\circ} \mathrm{STN}$ cell, filled with a chiral material. Using multilayer optics modeling, the director profiles in the cell at different times are analyzed and compared with predictions based on the Ericksen-Leslie theory. A few ms delay of the director change at the beginning of the switch has been found. A potential method for an increase in speed of some $25 \%$ of such liquid crystal devices is proposed.

The liquid crystal cell comprises two low index, indium tin oxide coated, glass plates, on top of which are surface aligning layers of rubbed polyimide. The plates are assembled together with $6.0 \mu \mathrm{m}$ spacers in between. The angle ${ }^{\text {a) }}$ Author to whom correspondence should be addressed; electronic mail:
yangfz@ mail.tsinghua.edu.cn between the rubbing directions of the upper and lower plates is $180^{\circ}$, forming a left-handed $180^{\circ}$ STN cell. The cell is filled with a liquid crystal mixture of ZLI-5100-000 and ZLI-5100-100, to which was also added a small quantity of a dopant ZLI-811 to help the formation of a uniform structure in the cell. The pitch of the material is $14.29 \mu \mathrm{m}$.

The cell is inserted between the two glass hemispheres, optical contact being achieved with matching fluid. This complete assembly is then placed so that the center of the sample is at the focus of the convergent laser beam. A schematic is given in Fig. 1. The He-Ne laser beam, of wavelength $632.8 \mathrm{~nm}$, is collimated into a less-coherent parallel beam of diameter about $5 \mathrm{~cm}$ by using diffusers and beam expander. ${ }^{10,11}$ This collimated beam passes through a polarizer, a horizontal slit aperture, and a pair of converging lenses, being focused to a spot at the center of the cell to be studied. Both reflectivity and transmissivity signals from the cell are recorded through a polarizer by a charge coupled device (CCD) camera (DALSA).

In order to obtain more sensitive data to the director profile in the cell it is first set such that the rubbing direction has an angle of about $45^{\circ}$ with the incident plane, and then we chose the incident angular region of the fully leaky guided modes from $60^{\circ}$ up to $78^{\circ}$ by setting the angle between the central axis of the convergent beam and the cell normal to be $70^{\circ}$. A $0.5 \mathrm{~ms}$ exposure time of the CCD cam-

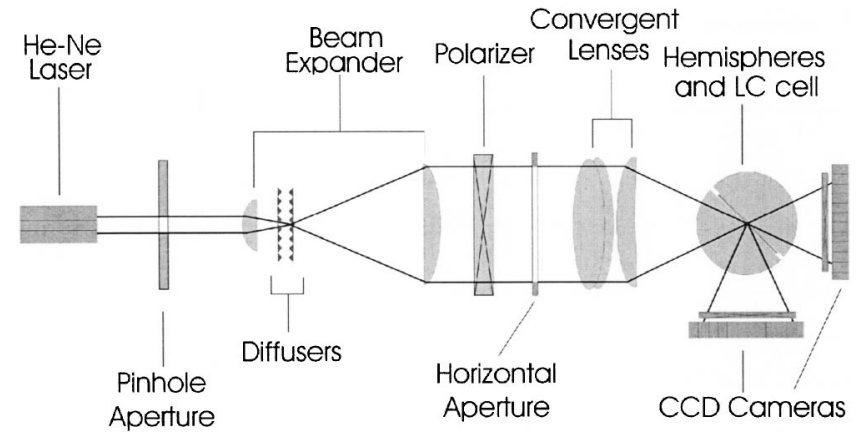

FIG. 1. The experimental setup for the convergent beam system. 

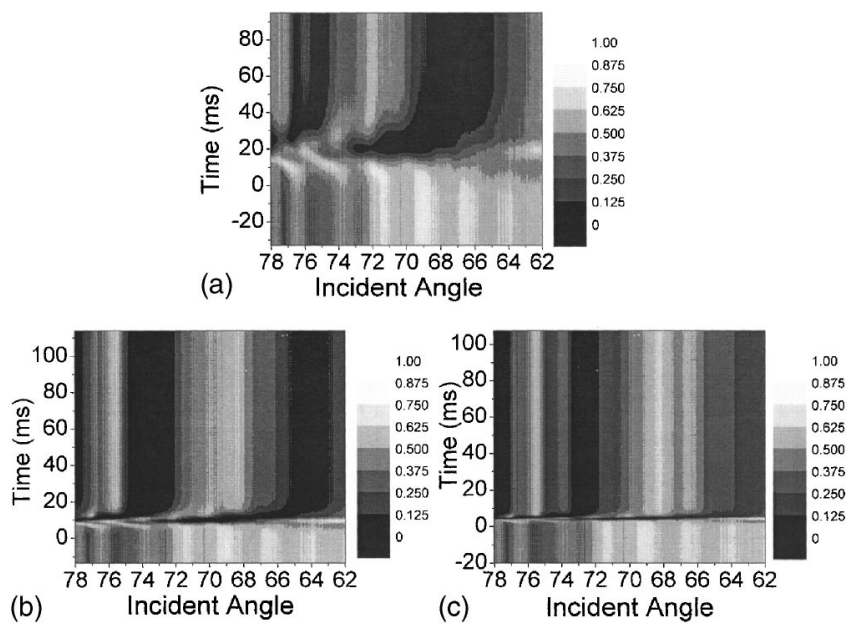

FIG. 2. The dynamic data of $T_{s s}$ after switch-on to (a) $2.8 \mathrm{~V}$, (b) $4.7 \mathrm{~V}$ and (c) $6.8 \mathrm{~V}(10 \mathrm{KHz})$.

era was chosen for taking high quality dynamic data (for details see Ref. 12).

By choice of input polarization setting ( $p$, transverse magnetic, or $s$, transverse electric) and output polarization setting eight data sets in total can be recorded ( $p$-to- $p$ and $s$-to- $s$ conserving and $p$-to-s and $s$-to- $p$ converting for both transmission and reflections). Data are first recorded with no voltage applied and then switch-on dynamic data are recorded under application of different ac $(10 \mathrm{kHz})$ fields of $2.8,4.7$, and $6.8 \mathrm{~V}$ (rms), respectively. Figure 2 shows how one of the recorded signals, $T_{s s}$, varies with time for the three different applied voltages. These figures show a dramatic change in the guided mode structure during switch-on. Switch-on times (to $\sim 95 \%$ final state) at voltages of $2.8,4.7$, and $6.8 \mathrm{~V}$ are about 60,20 , and $9 \mathrm{~ms}$, respectively.

Using multilayer optical theory ${ }^{13}$ to fit the reflection and transmission data, the full director profile and the material parameters of the different layers in the cell are obtained. For no applied field the optical parameters of the different layers are first found. The optical permittivities of the liquid crystal mixture are $\varepsilon_{\perp}=2.238+i 0.0005, \varepsilon_{\|}=2.694+i 0.0001$ with the thickness of the liquid crystal layer being $5.76 \mu \mathrm{m}$. The director uniformly twists through the whole cell from the top to the bottom through an angle of $180^{\circ}$ with surface tilts of about $1.0^{\circ}$ on both surfaces. The elastic constants and the dielectric permittivities of the liquid crystal mixture are obtained by fitting the data, taken after stabilization with the applied fields. Based on the Frank-Oseen elasticity theory, this gives $K_{11}, K_{22}$ and $K_{33}$ values of 12.84, 7.92, and $19.0 \mathrm{pN}$, respectively; the dielectric permittivities are $\varepsilon_{\perp}$ $=4.5, \varepsilon_{\|}=16.0$.

Having obtained all the static parameters of the cell, we analyze the dynamic data during switch-on by using a modeling program (DIMOS) based on the Berreman and van Doorn ${ }^{4,5}$ approximation of the Erickson-Leslie hydrodynamic theory. ${ }^{1-3}$ In this modeling the fluid inertia is ignored and flow is restricted to the plane of the cell. The dynamical data were fitted for all times by carefully selecting values for viscosity coefficients. Figure 3 shows the fit of the guided mode data, $T_{s s}$ and $T_{s p}$ at different times during switch-on to $6.8 \mathrm{~V}$. From the fit the values of the viscosity coefficients $\gamma$, $\eta_{1}, \eta_{2}, \eta_{3}$, and $\eta_{12}$ are also obtained. They are $0.120 \pm 0.002$, $0.170 \pm 0.004,0.018 \pm 0.001,0.04 \pm 0.01$, and $0.00 \pm 0.02$ Pas,
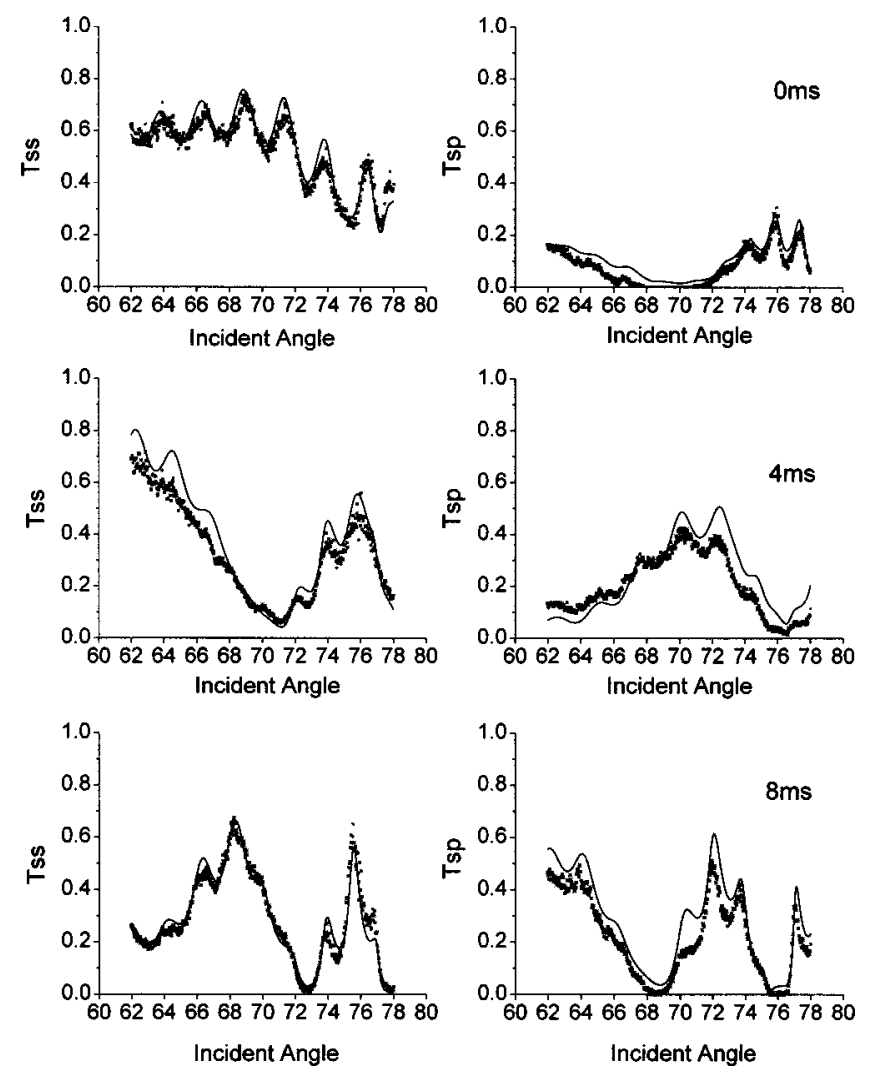

FIG. 3. Dynamic data $T_{s s}, T_{s p}$ (crosses) with the theoretical fits (solid line) at different times after switch-on of $6.8 \mathrm{~V}$ ac to the cell.

respectively. The rotational viscosity $\gamma$ plays the most important role.

From these fits the change of the director structure during the switch-on process are obtained. They are shown in Fig. 4 for different times after switching-on to $6.8 \mathrm{~V}$. It is found that at the beginning of the switch-on the behavior of the director twist is similar to that happening in a TN cell. ${ }^{14}$ During the initial $4 \mathrm{~ms}$ the director twists linearly in the cell, the same as the original state. However at $\sim 5 \mathrm{~ms}$ this twist profile suddenly changes to a dramatically different form. During intermediate times $(5-6 \mathrm{~ms})$ the director twists become concentrated near to the surface of the cell (as found for a $90^{\circ}$ twist cell ${ }^{14}$ ), and in a region of the cell center the director twist is uniform with only the director tilt varying. After $7 \mathrm{~ms}$ the director twist has a tendency to return to linearity. Then after about $9 \mathrm{~ms}$ the director twist profile changes to become much as that found in the cell with the steady applied field.

It is also worthwhile examining the change of the director tilt during the switch-on process. At the beginning of the
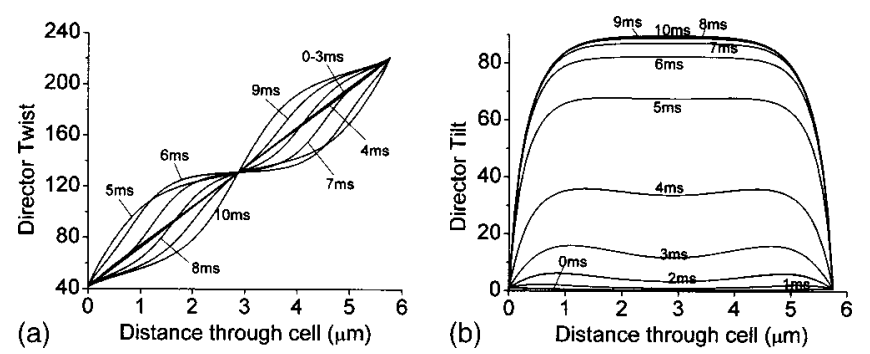

FIG. 4. Director profiles at different times after switch-on of $6.8 \mathrm{~V}$ ac to the cell. 


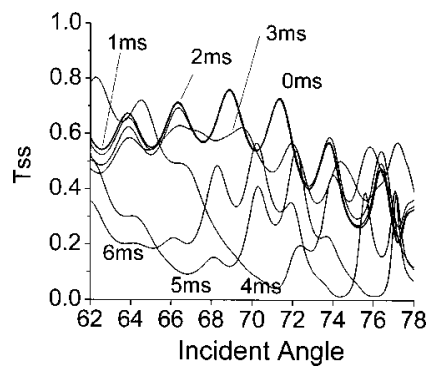

FIG. 5. Change of the fitted guided mode structure at different times after switch-on to $6.8 \mathrm{~V}$.

switch-on (within about $2 \mathrm{~ms}$ ) the director tilt undergoes little change (less than $3.5^{\circ}$ ). Correspondingly inspection of the fitted guided mode $\left(T_{s s}\right)$ structures (Fig. 5) shows clearly that within the first $2 \mathrm{~ms}$ there is only very small change in the guided mode structure. However, after some $2 \mathrm{~ms}$ the guided modes start to show significant change due to a big change in the director tilt (see Fig. 4). This means that the change of the liquid crystal structure mainly occurs between 3 and $5 \mathrm{~ms}$. Variation of the director tilt in the middle of the cell with time also shows this delay effect. This means that for this initial delay period the optical response of the cell is almost unchanged. There are approximately 2,4 , and $8 \mathrm{~ms}$ delays at the beginning for switching-on to $6.8,4.7$, and $2.8 \mathrm{~V}$, respectively. Comparing with the whole switch-on time this cannot be neglected.

If this delay can be eliminated, the switch-on will of course be faster. For example, if the cell is initially set in a small applied field (maybe $1.5 \mathrm{~V}$ ), instead of zero then, after switching to a higher voltage, the above delay would be removed, and the switch time is substantially reduced. From theoretical modeling for switching-on to 6.8 and $4.7 \mathrm{~V}$ through the cell the switch-on times would then be about 7 and $16 \mathrm{~ms}$, which is about $25 \%$ shorter. So this delay effect may be overcome to improve the switch-on speed of this type of liquid crystal device.

In conclusion, using the convergent beam system together with the fully leaky guided mode technique it has been possible to examine in detail the director switch-on process of an $180^{\circ} \mathrm{STN}$ cell. The approximation of Berreman and van Doorn of the Ericksen-Leslie theory accords well with the data taken from the cell. The switch-on process for different applied voltages has been analyzed and the voltage dependence of the response time explored. The behavior of the director twist in the STN cell during switch-on is similar to that of a $90^{\circ} \mathrm{TN}$ cell. It is found that the initiation of director tilt during the dynamical process causes a delay effect. This leads to a suggestion for speeding up such supertwisted devices.

This work was conducted under both the project 10174044 supported by NSFC and the Royal Society Joint UK-China Research Grant.

${ }^{1}$ J. L. Ericksen, Arch. Ration. Mech. Anal. 4, 231 (1960).

${ }^{2}$ J. L. Ericksen, Trans. Soc. Rheol. 5, 23 (1961).

${ }^{3}$ F. M. Leslie, Q. J. Mech. Appl. Math. 19, 357 (1966); 28, 265 (1968); Advances in Liquid Crystals, edited by G. H. Brown (Academic, New York, 1979), Vol. 4, p. 1.

${ }^{4}$ D. W. Berreman, J. Appl. Phys. 46, 3746 (1975).

${ }^{5}$ C. Z. van Doorn, J. Appl. Phys. 46, 3738 (1975).

${ }^{6}$ C. J. Gerritsma, C. Z. van Doorn, and P. van Zanten, Phys. Lett. 48A, 263 (1974).

${ }^{7}$ F. Nakano, H. Kawakami, H. Morishita, and M. Sato, Jpn. J. Appl. Phys. 19, 659 (1980).

${ }^{8}$ S.-H. Chen and C.-L. Yang, Appl. Phys. Lett. 80, 3721 (2002).

${ }^{9}$ O. Cossalter and D. A. Mlynski, Liq. Cryst. 19, 545 (1995).

${ }^{10}$ N. J. Smith and J. R. Sambles, J. Appl. Phys. 85, 3984 (1999).

${ }^{11}$ N. J. Smith and J. R. Sambles, Appl. Phys. Lett. 77, 2632 (2000).

${ }^{12}$ L. Ruan and J. R. Sambles, J. Appl. Phys. 92, 4857 (2002).

${ }^{13}$ D. Y. K. Ko and J. R. Sambles, J. Opt. Soc. Am. A 5, 1863 (1988).

${ }^{14}$ L. Ruan and J. R. Sambles, Phys. Rev. Lett. 90, 168701 (2003). 